\title{
Optimal Control Using Feedback Linearization for a Generalized T-S Model
}

\author{
Agustín Jiménez $^{1}$, Basil Mohammed Al-Hadithi ${ }^{1}$, Juan Pérez-Oria ${ }^{2}$, \\ and Luciano Alonso ${ }^{2}$
}

1 Intelligent Control Group, University Politecnica de Madrid, Centre for Automation and Robotics UPM - CSIC, Spain. C/ J. Gutierrez Abascal, 2. 28006-Madrid, Spain

www.disam.upm.es/control

2 University of Cantabria Santander, Spain

http://www.teisa.unican.es/gic/index.php

\begin{abstract}
In this paper, a fuzzy feedback linearization is used to control nonlinear systems described by Takagi-Suengo (T-S) fuzzy systems. In this work, an optimal controller is designed using the linear quadratic regulator (LQR). The well known weighting parameters approach is applied to optimize local and global approximation and modelling capability of T-S fuzzy model to improve the choice of the performance index and minimize it. The approach used here can be considered as a generalized version of T-S method. Simulation results indicate the potential, simplicity and generality of the estimation method and the robustness of the proposed optimal LQR algorithm.
\end{abstract}

\section{Introduction}

Feedback linearization has been used successfully to address some practical problems. These include the control of helicopters, high performance aircraft, industrial robots and biomedical devices. More applications of the methodology are being developed in industry 8 .

It is well known that a robust a effective controller requires an accurate and efficient model. In [9], an interesting method is developed to identify nonlinear systems using input-output data. The main problem encountered is that T-S identification method can not be applied when the MFs are overlapped by pairs.

Nonlinear control systems based on the T-S model have attracted lots of attention during the last twenty years (e.g., see [12, [5] and [6]). It provides a powerful solution for development of function approximation, systematic techniques to stability and design of fuzzy control systems in view of fruitful conventional control theory. They also allow relatively easy application of powerful learning techniques for their identification from data.

T-S fuzzy models are proved to be universal function approximators [1]. But it was clearly shown that the number of fuzzy rules increases as the approximation error tends to zero. So it becomes difficult to make use of the universal approximation property of T-S fuzzy modelling for practical purposes. Moreover, if the number of rules is bounded, the resulting set of functions is nowhere dense

L. Iliadis et al. (Eds.): AIAI 2014, IFIP AICT 436, pp. 466-475 2014.

(C) IFIP International Federation for Information Processing 2014 
in the space of approximated functions 10. These conflicting objectives have motivated researchers to find a balance between the specified accuracy and the computational complexity of the resulting fuzzy model.

In [1] and (7) new and efficient approaches are presented to improve the local and global estimation of T-S model. The first approach uses the minimum norm method to search for an exact optimum solution at the expense of increasing complexity and computational cost. The second one is a simple and less computational method, based on weighting of parameters.

The rest of the paper is organized as follows. A description of feedback linearization methodology is presented in section 2. In section 3 , the estimation of T-S fuzzy model is discussed. In section 4, the design of an optimal LQR controller is developed. In section 5, an example of an inverted pendulum is illustrated to demonstrate the validity of the proposed approach. The conclusions of the validity of the proposed estimation approach and the robustness of the LQR are explained in section 6.

\section{Feedback Linearization}

Feedback linearization is an approach to nonlinear control design which has attracted a great deal of research interest. The central ideal is to algebraically transform a nonlinear system dynamics into (fully or partly) linear one so that linear control techniqus can be applied. This differes entirely from conventional linearization (i.e. Jacobian linearization) in that feedback linearization is achieved by exact state transformation and feedback, rather than by linear approximations of the dynamics. Supposing that the system can be modeled as follows:

$$
y^{(n)}(t)=f_{n}\left(y(t), \dot{y}(t), \cdots, y^{(n-1)}(t)\right)+b_{n}\left(y(t), \dot{y}(t), \cdots, y^{(n-1)}(t)\right) u(t)
$$

Let us define the following vector:

$$
x=\left[y(t) \dot{y}(t) \cdots y^{(n-1)}(t)\right]^{T} \in \Re^{n}
$$

The system can be modeled as follows:

$$
\dot{x}_{n}(t)=f_{n}(x(t))+b_{n}(x(t)) u(t)
$$

For systems which can be expressed in the controllable and canonical form, the control action can be described as:

$$
u(x)=\frac{1}{b_{n}(x)}\left(s(x)-f_{n}(x)\right)
$$

Substituting (3) in (2) to obtain the feedback system:

$$
\dot{x}_{n}(t)=f_{n}(x)+b_{n}(x) u(t)=f_{n}(x)+b_{n}(x) \frac{1}{b_{n}(x)}\left(s(x)-f_{n}(x)\right)=s(x)
$$

Let us suppose the following control law:

$$
s(x)=S x=\left[\begin{array}{ll}
-s_{0}-s_{1} \cdots-s_{n-2}-s_{n-1}
\end{array}\right] x
$$


The feedback system becomes a linear one with a characteristic polynomial $p(\lambda)$ where the $s_{i}$ are chosen so that the the characteristic polynomial:

$$
p(\lambda)=\lambda^{n}+s_{n-1} \lambda^{n-1}+\cdots+s_{1} \lambda+s_{0}
$$

The first inconvenient is the need to know explicitly the nonlinear system model, which in general can not be obtained because of the difficulty of identifying their parameters. Secondly, an adequate choice of the characteristic polynomial should be made. A possible solution for the first problem is by using the T-S fuzzy model for estimating nonlinear systems.

$$
\begin{aligned}
S^{\left(i_{1} \ldots i_{n}\right)}: & \text { If } z_{1} \text { is } M_{1}^{i_{1}} \text { and } \ldots z_{f} \text { is } M_{n}^{i_{n}} \text { then } \\
\dot{x}= & a_{0}^{\left(i_{1} \ldots i_{n}\right)}+A_{n}^{\left(i_{1} \ldots i_{n}\right)} x+B_{n}^{\left(i_{1} \ldots i_{n}\right)} u
\end{aligned}
$$

where

$$
A_{n}^{\left(i_{1} \cdots \cdots i_{n}\right)}=\left[\begin{array}{llll}
a_{n 1}(x) & a_{n 2}(x) & \cdots & a_{n n}(x)
\end{array}\right]
$$

Applying this model, the identification of its parameters can be realized using the proposed estimation methods which will be described in section $[3$. We suppose that we have a first estimation of the T-S model parameters. In order to obtain such estimation, the classical least square method can be used around the equilibrium point. The objective is to obtain a local approximation of the system.

$$
\dot{x}_{n}=a_{o}^{0}+a_{n 1}^{0} x_{1}(x)+a_{n 2}^{0} x_{2}(x)+\cdots+a_{n n}^{0} x_{n}(x)+b_{n}^{0} u
$$

A design of an optimal controller is carried out to show the effectiveness of the proposed estimation methods. The well known LQR controller is applied to solve the second problem proposed in this work which is how to choose the optimal characteristic polynomial $p(\lambda)$ (see section 4).

\section{Estimation of Fuzzy T-S Model's Parameters}

An interesting method of identification is presented in [9]. The idea is based on estimating the nonlinear system parameters minimizing a quadratic performance index. It is based on the identification of functions of the following form:

$$
\begin{gathered}
f: \Re^{n} \longrightarrow \Re \\
y=f\left(v_{1}, v_{1}, \ldots, v_{n}\right)
\end{gathered}
$$

Each IF-THEN rule for an $n^{\text {th }}$ order system can be written as follows:

$$
\begin{aligned}
& S^{\left(i_{1} \ldots i_{n}\right)}: \text { If } v_{1} \text { is } M_{1}^{i_{1}} \text { and } \ldots v_{n} \text { is } M_{n}^{i_{n}} \text { then } \\
& \hat{y}=p_{0}^{\left(i_{1} \ldots i_{n}\right)}+p_{1}^{\left(i_{1} \ldots i_{n}\right)} v_{1}+p_{2}^{\left(i_{1} \ldots i_{n}\right)} v_{2}+\ldots+p_{n}^{\left(i_{1} \ldots i_{n}\right)} v_{n}
\end{aligned}
$$

where the fuzzy estimation of the output is:

$$
\hat{y}=\frac{\sum_{i_{1}=1}^{r_{1}} \ldots \sum_{i_{n}=1}^{r_{n}} w^{\left(i_{1} \ldots i_{n}\right)}(\mathbf{v})\left[p_{0}^{\left(i_{1} \ldots i_{n}\right)}+p_{1}^{\left(i_{1} \ldots i_{n}\right)} v_{1}+\ldots+p_{n}^{\left(i_{1} \ldots i_{n}\right)} v_{n}\right]}{\sum_{i_{1}=1}^{r_{1}} \cdots \sum_{i_{n}=1}^{r_{n}} w^{\left(i_{1} \ldots i_{n}\right)}(\mathbf{v})}
$$


where,

$$
w^{\left(i_{1} \ldots i_{n}\right)}(\mathbf{v})=\prod_{l=1}^{n} \mu_{l i_{l}}\left(v_{l}\right)
$$

being $\mu_{j i_{j}}\left(v_{j}\right)$ the membership function that corresponds to the fuzzy set $M_{j}^{i_{j}}$.

Let $\left\{v_{1 k}, v_{2 k}, \ldots, v_{n k}, y_{k}\right\}$ be a set of input/output system samples. The parameters of the fuzzy system can be calculated as a result of minimizing a quadratic performance index:

$$
J=\sum_{k=1}^{m}\left(y_{k}-\hat{y}_{k}\right)^{2}=\|Y-X P\|^{2}
$$

where

$$
\begin{aligned}
Y & =\left[\begin{array}{lll}
y_{1} & y_{2} \ldots y_{m}
\end{array}\right]^{T} \\
P & =\left[\begin{array}{llll}
p_{0}^{(1 \ldots 1)} & p_{1}^{(1 \ldots 1)} p_{2}^{(1 \ldots 1)} \ldots p_{n}^{(1 \ldots 1)} \ldots p_{0}^{\left(r_{1} \ldots r_{n}\right)} \ldots p_{n}^{\left(r_{1} \ldots r_{n}\right)}
\end{array}\right]^{T} \\
X & =\left[\begin{array}{llll}
\beta_{1}^{(1 \ldots 1)} \beta_{1}^{(1 \ldots 1)} v_{11} \ldots \beta_{1}^{(1 \ldots 1)} v_{n 1} \ldots \beta_{1}^{\left(r_{1} \ldots r_{n}\right)} \ldots \beta_{1}^{\left(r_{1} \ldots r_{n}\right)} v_{n 1} \\
\beta_{m}^{1 \ldots 1} & \beta_{m}^{(1 \ldots 1)} v_{1 m} \ldots \beta_{m}^{(1 \ldots 1)} v_{n m} \ldots \beta_{m}^{\left(r_{1} \ldots r_{n}\right)} \ldots \beta_{m}^{\left(r_{1} \ldots r_{n}\right)} v_{n m}
\end{array}\right]
\end{aligned}
$$

and

$$
\beta_{k}^{\left(i_{1} \ldots i_{n}\right)}=\frac{w^{\left(i_{1} \ldots i_{n}\right)}\left(\mathbf{v}_{k}\right)}{\sum_{i_{1}=1}^{r_{1}} \cdots \sum_{i_{n}=1}^{r_{n}} w^{\left(i_{1} \ldots i_{n}\right)}\left(\mathbf{v}_{k}\right)}
$$

If $X$ is a matrix of full rank, the solution is obtained as follows:

$$
\begin{aligned}
J & =\|Y-X P\|^{2}=(Y-X P)^{T}(Y-X P) \\
\nabla J & =X^{T}(Y-X P)=X^{T} Y-X^{T} X P=0 \\
P & =\left(X^{T} X\right)^{-1} X^{T} Y
\end{aligned}
$$

In the case when the matrix $\mathrm{X}$ is not of full rank, an effective approach with few computational effort, based on the well known parameters' weighting method [2], 1] and 7]. This method can also be used for parameters tuning of T-S model from local parameters obtained through the identification of a system in an operating region or from any physical input/output data. We suppose that we have a first estimation

$$
P_{0}=\left[p_{0}^{0} p_{1}^{0} p_{2}^{0} \ldots p_{n}^{0}\right]^{T}
$$

of the T-S model parameters. In order to obtain such an estimation, the classical least square method can be used around the equilibrium point. This first approximation can be utilized as reference parameters for all the subsystems. Then, the parameters' vector of the fuzzy model can be obtained minimizing:

$$
\begin{aligned}
J & =\sum_{k=1}^{m}\left(y_{k}-\hat{y}_{k}\right)^{2}+\gamma^{2} \sum_{i_{1}=1}^{r_{1}} \ldots \sum_{i_{n}=1}^{r_{n}} \sum_{j=0}^{n}\left(p_{j}^{0}-p_{j}^{\left(i_{1} \ldots i_{n}\right)}\right)^{2} \\
& =\|Y-X p\|^{2}+\gamma^{2}\left\|P_{0}-p\right\|^{2}=\left\|\left[\begin{array}{c}
Y \\
\gamma P_{0}
\end{array}\right]-\left[\begin{array}{c}
V \\
\gamma I
\end{array}\right] p\right\|^{2}=\left\|Y_{a}-V_{a} p\right\|^{2}
\end{aligned}
$$


where

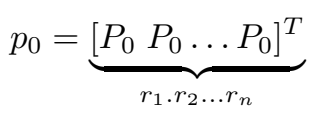

In this case, the factor $\gamma$ represents the degree of confidence of the parameters initially estimated [2], 3], 4] and [7].

\section{Design of an Optimal Controller}

A design of an optimal controller based LQR controller is applied to show the validity of the proposed estimation methods. The characteristic polynomial of the feedback system is calculated for this system so that finally, the parameters of this polynomial is used for the definition of $s(x)$. In order to calculate the control coefficients, any control design methodology through state feedback control can be applied.

Optimal selection of closed loop poles will lead to a trade-off between speed of dynamic response and control effort. Together with the proposed estimation method, LQR might be an appropriate choice. With this regulator we guarantee the stability and gaining a balance between static and dynamic behavior of the system with admissible control actions. Selection of values of $\mathrm{Q}$ and $\mathrm{R}$ matrices determines the dynamic speed of the controller as well as amplitudes of state variables and control signals.

$$
\begin{gathered}
\dot{x}=A x+B u \\
x \in \Re^{n}, u \in \Re^{m}, A \in \Re^{n \times n}, B \in \Re^{n \times m}
\end{gathered}
$$

The objective is to find the control action $\mathrm{u}(\mathrm{t})$ to transfer the system from any initial state $x\left(t_{0}\right)$ to some final state $x(\infty)=0$ in an infinite time interval, minimizing a quadratic performance index of the form:

$$
J=\int_{t_{0}}^{\infty}\left(x^{T} Q x+u^{T} R u\right) d t
$$

where $Q \in \Re^{n \times n}$ is a symmetric matrix, at least positive semidefinite one and $R \in \Re^{m \times m}$ is also a symetric positive definite matrix. The optimal control law is then computed as follows:

$$
\begin{aligned}
& u(t)=-K x(t) \\
& K=-R^{-1} B^{T} L
\end{aligned}
$$

where the matrix $L \in \Re^{n \times n}$ is a solution of the Riccati equation:

$$
0=-Q+L B R^{-1} B^{T} L-L A-A^{T} L
$$

The feedback system becomes:

$$
\dot{x}=A x+B u=A x-B K u=(A-B K) x
$$


and the characteristic polynomial is:

$$
p(\lambda)=\operatorname{det}(\lambda I-A+B K)
$$

It is aimed in this work to design an optimal LQR controller for the local approximation in (6). In order to obtain such estimation, the classical least square method can be used around the equilibrium point.

\subsection{Application of the Proposed Control Algorithm for T-S Systems}

The new proposed control algorithm can be considered as a two level one: the first level includes the calculation of the resulting matrices $a_{0}(x), A(x)$ and $B(x)$ from the above identified fuzzy system and the second one is obtaining the control action mentioned above in (3). The control law becomes:

$$
u(x)=\frac{1}{b_{n}(x)}\left(S X-a_{0 n}(x)-A_{n}(x) x\right)=-\frac{1}{b_{n}(x)}\left(a_{0 n}(x)+\left(A_{n}(x)-V\right) x\right)
$$

\section{Illustrative Example}

Consider the problem of estimating an inverted pendulum using the above mentioned estimation methods. The inverted pendulum can be represented as follows:

$$
\ddot{\theta}=\frac{g \sin \theta-\cos \theta\left(\frac{u+m l \dot{\theta}^{2} \sin \theta}{M+m}\right)}{l\left(\frac{4}{3}-\frac{m \cos ^{2} \theta}{M+m}\right)}
$$

Where $\theta$ denotes the angular position (in radians) deviated from the equilibrium position (vertical axis) of the pendulum and $\dot{\theta}$ is the angular velocity, $\mathrm{g}$ (gravity acceleration), $\mathrm{M}$ (mass) of the cart $=1 \mathrm{~kg}, \mathrm{~m}$ (mass) of the pole $=0.1 \mathrm{~kg}$, $l$ is the distance from the center of the mass $(\mathrm{m})$ of the pole to the cart $=0.5$ $\mathrm{m}$. Assuming that $\mathrm{x}_{1}=\theta$ and $\mathrm{x}_{2}=\dot{\theta}$, then (17) can be rewritten in state space form as follows:

$$
\begin{aligned}
& x_{1}=\theta \\
& \dot{x}_{1}=x_{2} \\
& x_{2}=\dot{\theta} \\
& \dot{x}_{2}=\frac{g \sin \left(x_{1}\right)-\cos \left(x_{1}\right)\left(\frac{u+m l x_{2}^{2} \sin \left(x_{1}\right)}{M+m}\right)}{l\left(\frac{4}{3}-\frac{m \cos ^{2}\left(x_{1}\right)}{M+m}\right)}
\end{aligned}
$$

Firstly, the model of the inverted pendulum is estimated in three operation points for both the angle and its derivative. The universe of discourse of the angle is $\left[\frac{-\pi}{4}, \frac{\pi}{4}\right] \mathrm{rad}$. and the one of the angular velocity is $[-5,5] \frac{\mathrm{rad}}{\mathrm{seg}}$. Both MFs for the angle $\mathrm{x}$ and its derivative $\dot{x}$ are shown in figures $11 \mathrm{y} 2$ respectively.

If we apply the T-S method directly to this example, then the condition number of the matrix $\mathrm{X}$ is $3.4148 \mathrm{e}+015$, which shows clearly a non reliable result. 


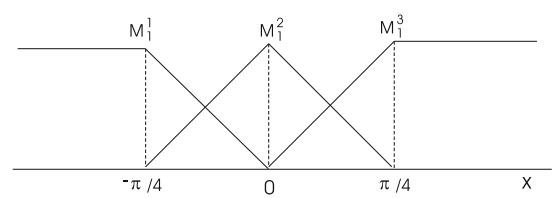

Fig. 1. Membership functions for the angle $x$ of the inverted pendulum

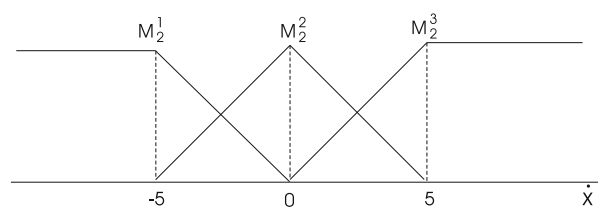

Fig. 2. Membership functions for the angular velocity of the inverted pendulum

Using the parameters' weighting method with weighting factor $\gamma=0.01$, the inverted pendulum fuzzy model can be represented as follows:

$$
\begin{aligned}
S^{11} & : \text { If }\left(x_{1} \text { is } M_{1}^{1}\right) \text { and }\left(x_{2} \text { is } M_{2}^{1}\right) \text { then } \\
\dot{x}_{2} & =-8.2354+3.3866 x_{1}-0.2040 x_{2}-1.0443 u \\
S^{12} & : \text { If }\left(x_{1} \text { is } M_{1}^{1}\right) \text { and }\left(x_{2} \text { is } M_{2}^{2}\right) \text { then } \\
\dot{x}_{2} & =-8.3313+3.0983 x_{1}+0.0000 x_{2}-1.0443 u \\
\vdots & \\
S^{33} & : \text { If }\left(x_{1} \text { is } M_{1}^{3}\right) \text { and }\left(x_{2} \text { is } M_{2}^{3}\right) \text { then } \\
\dot{x}_{2} & =8.0784+3.8914 x_{1}-0.2297 x_{2}-1.0903
\end{aligned}
$$

By using the identification with the classical minimum square method in an interval close to the equlibrium point

$$
x_{1} \in\left[\frac{-\pi}{4}, \frac{\pi}{4}\right] \quad \mathrm{x}_{2} \in[-2.5,2.5]
$$

The linear model of the system in this interval is:

$$
\dot{x}_{2}=0.0092+15.2665 x_{1}-0.0000 x_{2}-1.4187 u
$$

As it has been mentioned before, the inestable equilibrium point which will be also the objective of control is $x_{1}=x_{2}=u=0$. In order that this point becomes an equlibrium one in the fuzzy model, $a_{0}^{22}$ should be zero. The resultant mean square error from this approximation is 0.0013 . In this case, the condition number of the extended matrix $X_{a}$ becomes $1.4569 \mathrm{e}+004$, thus improving the reliability of results.

As it can be observed, the results obtained through the parameters weighting method are always better than with the original T-S method. In fact, the proposed method correspond to the best possible result using TS method when the interval covering tends to totality, but this limit is not achievable since the problem would not be solvable any more.

Consider the problem of stabilizing and balancing of swing up of an inverted pendulum. The control of this system is a widely used performance measure of a controller, since this system is unstable and highly nonlinear. The objective is to maintain the inverted pendulum upright with $\theta$ despite small disturbances due 
to wind or system noises. The proposed optimum LQR is applied to calculate the desired parameters of the characteristic polynomial $p(\lambda)$ defined in the previous rules,

$$
\left[\begin{array}{l}
\dot{x}_{1} \\
\dot{x}_{2}
\end{array}\right]=\left[\begin{array}{cr}
0 & 1 \\
15.2665 & 0
\end{array}\right]\left[\begin{array}{l}
x_{1} \\
x_{2}
\end{array}\right]+\left[\begin{array}{c}
0 \\
-1.4187
\end{array}\right] u
$$

minimizing the following performance index:

$$
J=\int_{t_{0}}^{\infty}\left(100 x_{1}^{2}+10 x_{2}^{2}+u^{2}\right) d t
$$

and the computed control becomes

$$
u(t)=-K x(t)=[25.45096 .7734] x(t)
$$

The matrix of the feedback system becomes:

$$
A_{R}=A-B K=\left[\begin{array}{cc}
0 & 1 \\
-20.8408 & -9.6095
\end{array}\right]
$$

and its characteristic polynomial is:

$$
p(\lambda)=\lambda^{2}+9.6095 \lambda+20.8408
$$

and the eigen values are:

$$
\lambda_{1}=-6.3029 \quad \lambda_{2}=-3.3065
$$

Thus, the control becomes:

$$
u(x)=\frac{1}{b_{n}(x)}\left(a_{0 n}(x)+\left(A_{n}(x)-S\right) x\right)
$$

where:

$$
S=[-20.8408-9.6095]
$$

Figure 3 shows the transient response of the inverted pendulum controlled by the proposed LQR subjected to an initial condition. The results obtained show that the system is stabilized by applying the proposed LQR.

Moreover, we have proven that the LQR is robust and invariant against measurement noise. If we suppose that the angle sensor introduces a noise of $\sigma=1^{\circ}$, it can be observed that the output is only affected by $\sigma=0.04^{\circ}$ as shown in figure 4. Figure [5]shows several trajectories in state space form of the system for several initial conditions. 


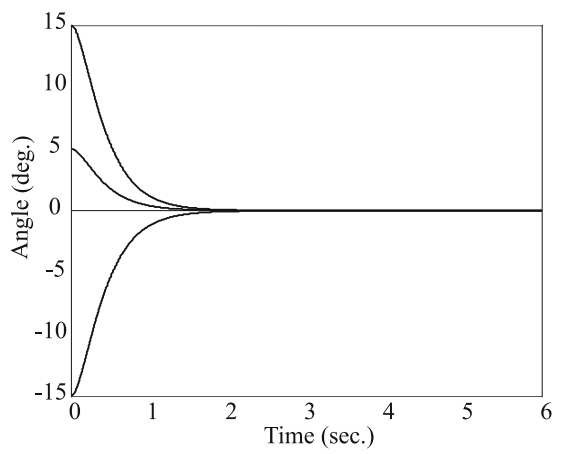

Fig. 3. Transient response of the inverted pendulum by the fuzzy LQR

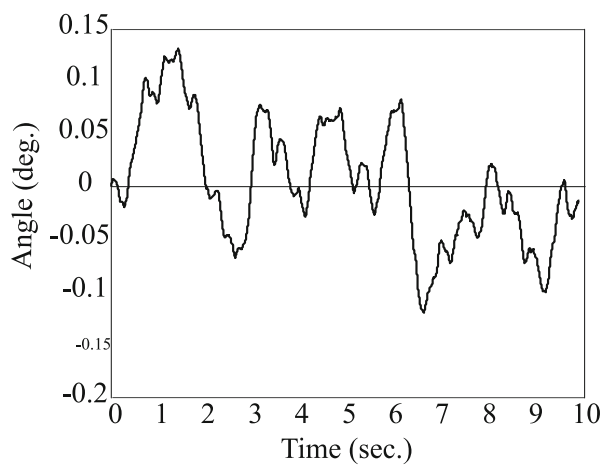

Fig. 4. Transient response of the inverted pendulum by the fuzzy LQR subjected to measurement noise

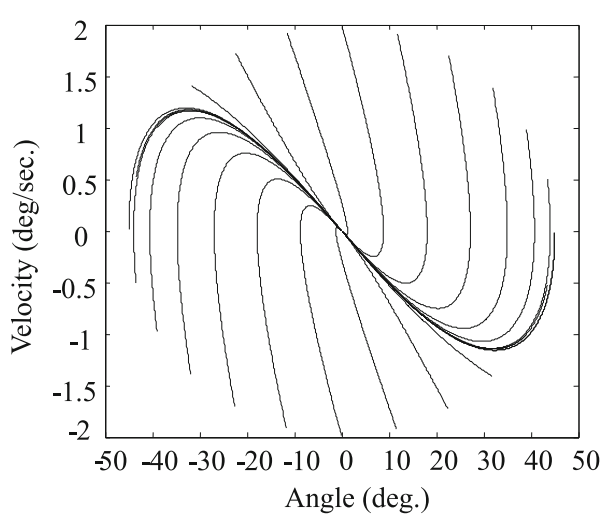

Fig. 5. Trajectories in state space form of the system for several initial conditions

\section{Conclusions}

A feedback linearization is used to nonlinear control design for fuzzy systems described by T-S. The main objective is to obtain an improved performance of highly non-linear unstable systems. In this work, the well known weighting parameters approach is proposed to optimize local and global approximation and modelling capability of T-S fuzzy model to improve the choice of the performance index and minimize it. An inverted pendulum mounted on a cart is chosen to evaluate the robustness, effectiveness, accuracy and remarkable performance of proposed estimation approach. The results show that the proposed approach is less conservative than those based on (standard) T-S model. Simulation results indicate the potential, simplicity and generality of the estimation method and the robustness of the LQR algorithm. We prove that the proposed estimation algorithm converge very fast, thereby making them very practical to use. 
Acknowledgements. This work is funded by Spanish Ministry of innovation and Science (ARABOT project DPI2010-21247-C02-01).

\section{References}

1. Al-Hadithi, B.M., Jiménez, A., Matía, F.: New Methods for the Estimation of T-S Model Based Extended Kalman Filter and its Applications to Optimal Control for Nonlinear Systems. Optimal Control, Applications and Methods 33(5), 552-575 (2012)

2. Al-Hadithi, B.M., Jiménez, A., Matía, F.: Variable Structure Control with Chattering Reduction of a Generalized T-S Model. Asian Journal of Control 15(1), 1-14 (2013)

3. Al-Hadithi, B.M., Barragán, A.J., Andújar, J.M., Jiménez, A.: Variable Structure Control with chattering elimination and guaranteed stability for a generalized T-S model. Applied Soft Computing 13, 4802-4812 (2013)

4. Al-Hadithi, B.M., Jiménez, A., Matía, F.: A new approach to fuzzy estimation of Takagi-Sugeno model and its applications to optimal control for nonlinear systems. Applied Soft Computing 12, 280-290 (2012)

5. Gang, F.: A survey on analysis and design of model-based fuzzy control systems. IEEE Trans. Fuzzy Syst. 14(5), 676-697 (2006)

6. Hseng, T., Li, S., Tsai, S.-H.: Fuzzy Bilinear Model and Fuzzy Controller Design for a Class of Nonlinear Systems. IEEE Trans. Fuzzy Syst. 15(3), 494-506 (2007)

7. Jiménez, A., Al-Hadithi, B.M., Matía, F.: Improvement of Takagi-Sugeno Fuzzy Model for the Estimation of Nonlinear Functions. Asian Journal of Control 14(2), 320-334 (2012)

8. Slotine, J.-J.E., Li, W.: Applied nonlinear control. Prentice Hall (1991) ISBN: 013-040890-5

9. Takagi, T., Sugeno, M.: Fuzzy identification of systems and its applications to modeling and control. IEEE Trans. Syst., Man, Cybern. SMC-15(1), 116-132 (1985)

10. Tikk, D., Baranyi, P., Patton, R.J.: Polytopic and TS Models are Nowere Dense in the Approximation Model Space. In: Proc. IEEE Int. Conf. Syst. Man Cybern. Methodology,

11. Zeng, K., Zhang, N.Y., Xu, W.L.: A comparative study on sufficient conditions for Takagi-Sugeno fuzzy systems as universal approximators. IEEE Trans. Fuzzy Syst. 8(6), 773-780 (2000)

12. Barragán, A.J., Al-Hadithi, B.M., Jiménez, A., Andújar, J.M.: A general methodology for online TS fuzzy modeling by the extended Kalman filter. Applied Soft Computing 18, 277-289 (2014) 